\title{
Multi-windows Threshold-based Frame Synchronization in Variable Coded Modulation Service
}

\author{
Yong Sheng , Jinshu Chen and Xiaoming Fan \\ Department of Electronic Engineering, 100084 Tsinghua University, Beijing, China
}

\begin{abstract}
In this paper, an innovative and reliable frame synchronization scheme is proposed for second generation digital video broadcasting (DVB-S2) system in Variable Coded Modulation (VCM) service. Frame synchronization in VCM suffers many difficulties from large frequency offset, extremely low SNR and variable physical layer frame (PLFRAME) lengths. To cope with variable PLFRAME lengths, multi-windows are proposed and utilized. Multiwindows threshold-based search algorithm is presented with theoretical analysis to decrease the missed detection probability. Experimental results demonstrate that our proposed method is more robust and reliable than the existing approaches, especially in low signal to noise ratios (SNRs) and large frequency offsets.
\end{abstract}

\section{Introduction}

Second generation digital video broadcasting (DVB-S2) via satellites has been standardized for a decade. It draws much attention since its distinctive transmission performance could approach the Shannon limit. Compared with DVB, DVB-S2 provides Adaptive Coded Modulation (ACM) and Variable Coded Modulation (VCM), implying that channel coding and modulation could be changed on a frame-by-frame basis [1]. VCM makes full use of the channel by transmitting more information bits when signal-to-noise ratio (SNR) is high, e.g., uses 9/10 LDPC and 32APSK combination, and makes sure of receiver performance by throttling down the information rate when SNR is low, e.g., exploits $1 / 2$ LDPC and QPSK combination. This flexible character results in a much higher total throughput than that obtained with constant coded modulation (CCM).

Even though VCM can provide an obvious improvement in achievable throughput over CCM, with nearly 1.7 times [2], it suffers many difficulties from frame synchronization, because of large frequency offset, extremely low SNR and variable physical frame lengths.

Many algorithms on frame synchronization have been researched and recommended. Based on the approximate maximum likelihood (ML) criterion, Choi and Lee proposed a differential detector in [3], known as CLD, which is insensitive to large frequency offset but has high complexity. NCPDI (Non-Coherent post detection integration) [4] and DPDI (Differential post detection integration) [5] are studied to decrease implementation complexity, while they are susceptible to unknown frequency offset. ETSI recommends a scheme in [6] to correlate on the SOF and PLSCODE differentially, which can work smoothly in large frequency offset and has comparatively low complexity.

After obtaining differential correlation, traditionally, decisions can be made simply by comparing correlation with a pre-set threshold [6]. Frame synchronization is deemed as achieved if the value is higher than the threshold. On account of inflexible threshold setting, peak detector is introduced in [7] to get rid of threshold. To strengthen the robustness of non-threshold scheme, double peak search algorithm is discussed in $[8,9]$.

There are, however, few investigations into frame synchronization in VCM application. Variable frame lengths make it hard to detect physical frame header (PLHEADER). How to ensure less frame synchronization drops in coding and modulation (CODMOD) switchovers is another issue to be solved. In addition, the performances of existing methods for frame synchronization are unsatisfactory at low SNRs. In this paper, multi-windows threshold-based search algorithm is developed to cope with these problems. Our simulation results indicate that the proposed approach can acquire satisfactory performance than existing methods.

The rest of this paper is organized as follows. In section 2, the structure and features of DVB-S2 physical frame are analysed. Then, VCM service is discussed in section 3, with description of the structure of VCM transmitter and receiver. Moreover, the complete frame synchronization schemes are proposed in detail in section 4 , including differential detection module and multiwindows threshold-based algorithm. Some optimizations and comparisons of the scheme are discussed in section 5 . Section 6 shows the performance of MATLAB simulation. Finally, conclusions are drawn in section 7. 


\section{DVB-S2 frame structure analysis}

\subsection{Physical layer frame structure}

According to DVB-S2, Physical Layer Header (PLHEADER) occupies one slot (90 symbols) in Physical Layer (PL), consisting of Start of Frame (SOF) and Physical Layer Signalling Code (PLSC). SOF corresponds to 26-symbol fixed pseudo-random pattern (18D2E82), which assists in frame synchronization and carrier recovery. PLSC is a 64-symbol linear binary code, conveying seven bits of information (e.g., coding, modulation type, pilot insertion and frame length) by RM transformation. To make receiver synchronization easier and simpler, pilot blocks could insert every 16 slots. The pilot block is made up of 36 un-modulated symbols, which shall be utilized in section 5 to enhance performance. Fig. 1 shows the structure of PLFRAME.

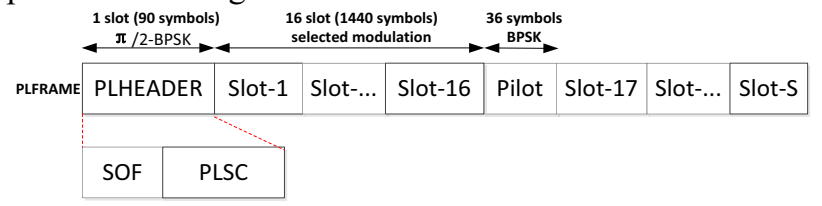

Figure 1. PLFRAME structure.

In VCM service, different frames can adopt optimal coding and modulation mode (CODMOD) to take advantage of dynamic link. Fig. 2 gives an example of variable CODMODs in continuous frames.

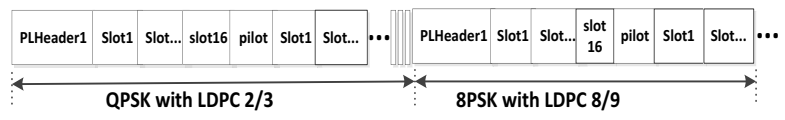

Figure 2. Example of VCM.

\subsection{Variable PLFRAME lengths}

According to [1], Forward Error Correction (FEC) Encoding is implemented by the concatenation of $\mathrm{BCH}$ outer code and LDPC (Low Density Parity Check) inner code. After FEC coding, the length of coded block shall be 64800 bits or 16200 bits, depending on the specific application field. When VCM is on service, FEC and modulation mode could vary in different frames, while they are kept constant in all slots of one frame. Modulation set contains QPSK, 8PSK, 16APSK and 32APSK. The length of PLFRAME can be obtained in table 1 , which determines the width of window in section 4.

Table 1. Frame Parameters.

\begin{tabular}{|c|c|c|c|c|c|}
\hline \multicolumn{2}{|c|}{ FECFRAME } & \multicolumn{4}{c|}{ PLFRAME } \\
\hline $\begin{array}{c}\text { Frame } \\
\text { length } \\
\text { (bits) }\end{array}$ & $\begin{array}{c}\text { Mod } \\
\text { type }\end{array}$ & Slot & $\begin{array}{c}\text { Pilot } \\
\text { block }\end{array}$ & $\begin{array}{c}\text { Has } \\
\text { Pilot }\end{array}$ & $\begin{array}{c}\text { PLFRAME } \\
\text { length } \\
\text { (bauds) }\end{array}$ \\
\hline 64800 & QPSK & 360 & 22 & Yes & 33282 \\
\hline 64800 & QPSK & 360 & 22 & No & 32490 \\
\hline 64800 & 8PSK & 240 & 14 & Yes & 22194 \\
\hline 64800 & 8PSK & 240 & 14 & No & 21690 \\
\hline 64800 & 16 APSK & 180 & 11 & Yes & 16686 \\
\hline
\end{tabular}

\begin{tabular}{|c|c|c|c|c|c|}
\hline \multicolumn{2}{|c|}{ FECFRAME } & \multicolumn{4}{c|}{ PLFRAME } \\
\hline $\begin{array}{c}\text { Frame } \\
\text { length } \\
\text { (bits) }\end{array}$ & $\begin{array}{c}\text { Mod } \\
\text { type }\end{array}$ & Slot & $\begin{array}{c}\text { Pilot } \\
\text { block }\end{array}$ & $\begin{array}{c}\text { Has } \\
\text { Pilot }\end{array}$ & $\begin{array}{c}\text { PLFRAME } \\
\text { length } \\
\text { (bauds) }\end{array}$ \\
\hline 64800 & 16 APSK & 180 & 11 & No & 16290 \\
\hline 64800 & 32 APSK & 144 & 8 & Yes & 13338 \\
\hline 64800 & 32 APSK & 144 & 8 & No & 13050 \\
\hline 16200 & QPSK & 90 & 5 & Yes & 8370 \\
\hline 16200 & QPSK & 90 & 5 & No & 8190 \\
\hline 16200 & 8 PSK & 60 & 3 & Yes & 5598 \\
\hline 16200 & 8 PSK & 60 & 3 & No & 5490 \\
\hline 16200 & 16 APSK & 45 & 2 & Yes & 4212 \\
\hline 16200 & 16 APSK & 45 & 2 & No & 4140 \\
\hline 16200 & 32 APSK & 36 & 2 & Yes & 3402 \\
\hline 16200 & 32 APSK & 36 & 2 & No & 3330 \\
\hline
\end{tabular}

\section{VCM service design}

Many satellite missions communicate in a highly dynamic environment, due to the variations in geometry, weather and interference [10]. To make best use of link margin when channel condition is good, high order coding and modulation (CODMOD) is preferred to obtain maximum throughput. In order to enable communication in poor channel, low order CODMOD is considered to guarantee the receiver performance. VCM service shall be designed to meet this requirement. Even though CODMOD could vary on frame basis, too many switchovers between CODMODs shall take down the overall performance contrarily for expensive overheads. According to [2], orbits are divided into a fixed number (e.g., 6) of sectors (geometric approach), and link budgets are computed in each sector according to local worst conditions.

In VCM service, the transmitter structure can be designed as Fig. 3. Several minutes before transmission mission, VCM system shall select certain sequence of CODMODs (e.g., 5 or 6) according to channel condition and link budget. During satellite passing, transmission system on the satellite shall follow presupposed CODMODs to process information frame on sector basis.

Receiver structure is showed in Fig. 4, where frame synchronization is in dashed box, which is the concern of this paper.

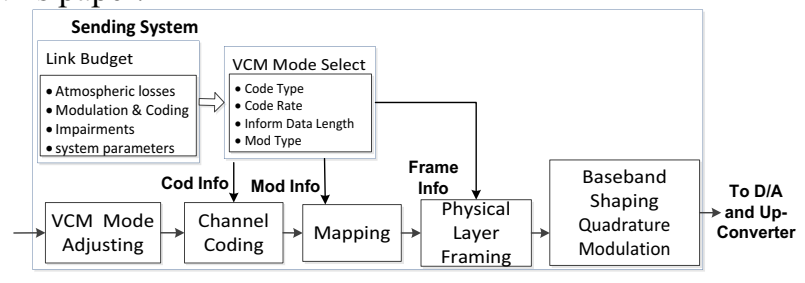

Figure 3. Transmitter structure of VCM.

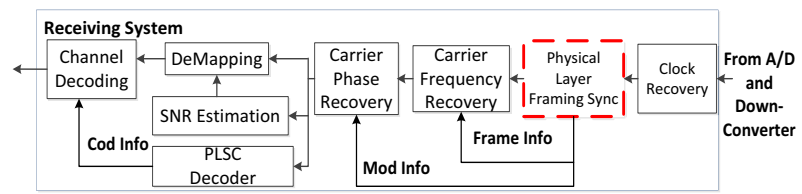

Figure 4. Receiver structure of VCM. 


\section{Proposed frame synchronization scheme}

As shown in Fig. 4, after the steady convergence of receiver symbol timing, frame synchronization for PLHEADER shall be in place. Generally speaking, frame synchronization consists of acquisition and tracking. Frame acquisition is the process of calculating differential correlation, which is performed by differential detector. PLHEADER tracking is the phase to determine the accurate location of PLHEADER and modulation type in the current frame. To deal with variable frame lengths, multi-windows are utilized. An innovative and reliable method based on threshold is proposed to achieve satisfactory performance, especially in the situation of extremely low SNR and large frequency offset.

\subsection{Differential detector}

ETSI recommends a scheme in [6] to correlate on the SOF and PLSCODE differentially, which can work smoothly in the presence of large frequency offsets $(20 \%$ of the symbol transmission rate) and low SNR $(-2.35 \mathrm{~dB})$. The differential correlation $\Lambda$ can be achieved in equation

$$
\wedge=\left|\sum_{k=1}^{25} r_{k} r_{k+1}^{*} C_{S O F k}^{*}+\sum_{m=1}^{45} R_{2 m-1} R_{2 m}^{*} C_{P L S C m}^{*}\right|
$$

where $r$ denotes the received symbol of SOF, $R$ is the received symbol of PLSC, $C_{S O F}$ and $C_{P L S C}$ are the corresponding differential coefficients obtained for SOF and PLSC respectively, and star mark represents complex conjugate. $C_{S O F}$ and $C_{P L S C}$ can be obtained by equation

$$
\begin{aligned}
& C_{S O F k}=s_{k} s_{k+1}^{*}= \pm j, k=1,2, \ldots, 24,25, \\
& C_{P L S C m}=s_{2 m-1} s_{2 m}^{*}= \pm j, m=1,2, \ldots, 44,45,
\end{aligned}
$$

where $s$ is the transmitted PLHEADER symbol.

The diagram of differential correlation circuit is shown in Fig. 5.

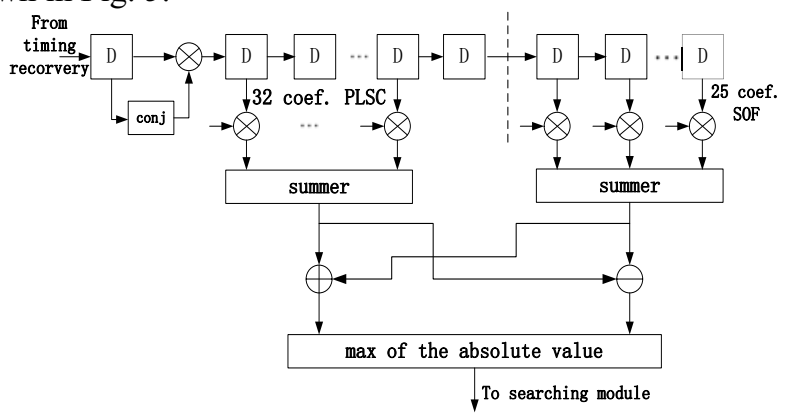

Figure 5. Differential detection circuit of the PLHEADER.

\subsection{Multi-windows threshold-based search algorithm}

The differential output is then sent to a peak search module to track the PLHEADER. The conventional peak search module simply compares the output of correlator with a predefined threshold [6]. In [7], peak search algorithm with one constant width window is raised to get rid of threshold setting. To decrease missed detection probability in poor channel condition, [8] suggests a double peak search method, choosing two maximum peaks in one window as the candidates. However, no matter one peak, double peaks or more, the missed detection probability is rather high when SNRs below $1 \mathrm{~dB}$. Moreover, to the best of our knowledge, none of the existing studies considers the VCM frame synchronization clearly and correctly, which shall be the crucial concern of this section.

As table 1 describes, 16 types of frame length are available in VCM system. Since there is one-to-one correspondence relationship between modulation and pilot status with frame length, frame length information can be achieved by comparing two neighbour PLHEADER positions. Without prior information about CODMODs in receiver, 16 types of search windows with corresponding widths shall be in consideration.

Without loss of generality, it is supposed that only 4 types of frame exist, QPSK, 8PSK, 16APSK and 32APSK with pilot insertion, and their corresponding widths are 13338, 16686, 22194 and 33282. As Fig. 6 shows, symbol differential correlations in 5 physical layer frames at $\mathrm{SNR}=-2.35 \mathrm{~dB}$ are in hands. The five red bold lines represent the differential correlations of PLHEADER symbols. PHLEAHER symbol represent the first symbol of PLHEADER. Then, all of symbols whose differential correlations are above threshold 0.77 shall be in further investigation. In Fig. 7, any symbol with its differential correlation higher than 0.77 is recorded.

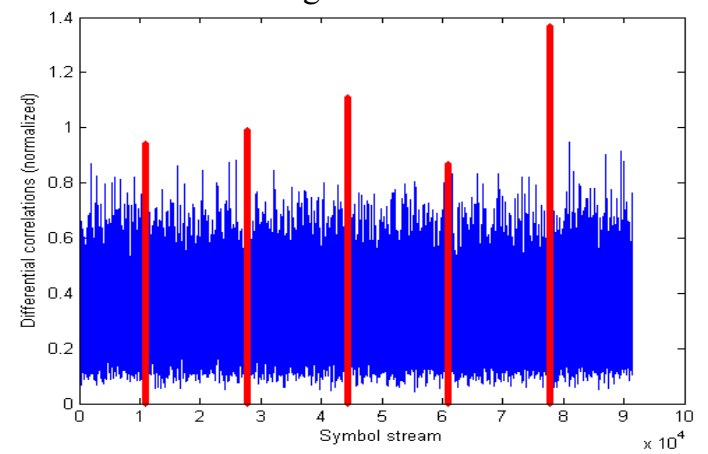

Figure 6. Symbol Differential correlations of 5 frames at $\mathrm{SNR}=-2.35 \mathrm{~dB}$.

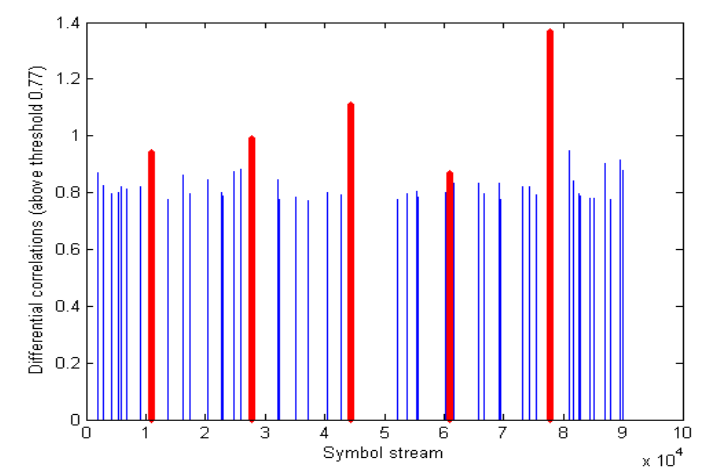

Figure 7. Symbol Differential correlations that are above 0.77 .

Afterwards, position comparison is made within three successive search windows. As is shown in Fig. 8, the 
first search window corresponds to 32APSK with pilot insertion. Dashed boxes denote window inspection sections. If any two positions that relative to the left end of their own search window share the same value, the position is immediately deemed as aligned to the PLHEADER, and the search window shall slide to new place to start a new round of PLHEADER detection. If none can match in this kind of three successive windows, similar operations are carried on within next kind search window, which corresponds to 16APSK with pilot insertion. As is shown in Fig. 8, in the second type of search window, three positions relative to their own search window are quite same, indicating that PLHEDER is obtained at this position and the modulation of this PLFRAME is 16APSK.

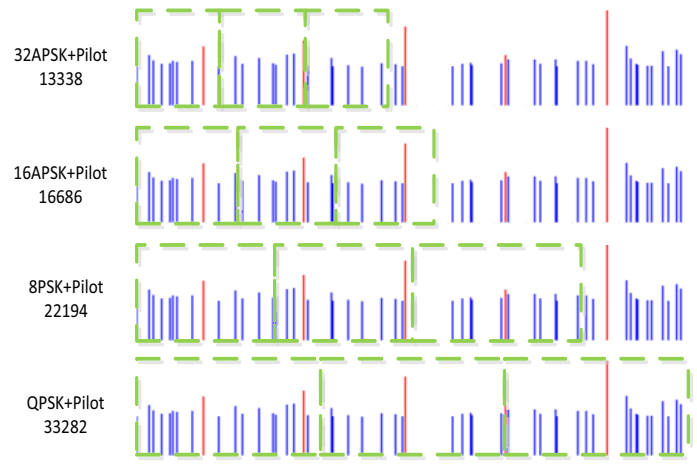

Figure 8. Multi-windows threshold-based algorithm processing on 5 frames.

Obviously, the value of threshold has a great influence on frame synchronization probability. In the next part, the relationship between threshold and frame synchronization probability shall be investigated.

Without noise interference, the differential correlation of PLHEADER symbol is 57—-sum of PLSC differential correlation 32 and SOF differential correlation 25. Considering the poor condition with extremely low $\mathrm{SNR}=-2.35 \mathrm{~dB}$ and large frequency offset of $20 \%$ of transmitting rate, some simulations over performance of differential correlation detector are conducted. In Fig. 9, PLHEADER symbol refers to the first symbol of PLHEADER, and non-PLHEADER symbols refer to other symbols except from PLHEADER symbol in a PLFRAME. All symbol differential correlations are normalized to 1 by dividing 57 .

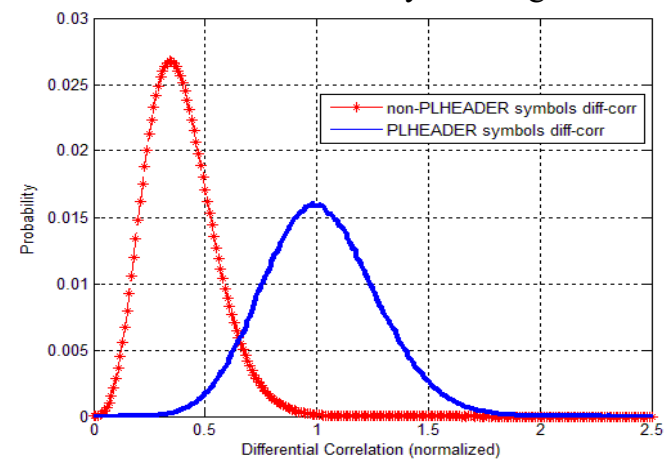

Figure 9. Symbol differential correlation distribution curve.

From Fig. 9, some hidden relationships between frame synchronization probability and false alarm probability with threshold could be discovered. Let $T$ represent some certain threshold, $P_{\text {header }}(t)$ represents the blue solid line_PLHEADER symbol differential correlation distributions, and $P_{\text {non-header }}(t)$ denotes the dashed line with star marker $\longrightarrow$ non-PLHEADER symbol differential correlations distribution. $P_{+}$ represents the probability that PLHEADER symbol differential correlation is higher than threshold. And $P$ refers to the probability of non-PLHEADER being below threshold.

$$
\begin{aligned}
& P_{+}=\int_{T}^{+\infty} P_{\text {header }}(t) d t, \\
& P_{-}=\int_{0}^{T} P_{\text {non-header }}(t) d t .
\end{aligned}
$$

If two candidates in three successive window share the same relative position in terms of their belonging windows, then this position can be deemed as PLHEADER. Supposing that differential correlations in different windows are independent identically distributed, the ideal synchronization rate can be get from the equation

$$
P_{\text {synched }}=P_{+}^{3}+C_{3}^{2} P_{+}^{2}\left(1-P_{+}\right)
$$

Actually, the synched rate shall be lower than $P_{\text {synched, }}$ for interfere of false PLHEADER symbols. If two relative positions of non-PLHEADER symbols exactly equal in continuous three frame, false synched PLHEADER occurs, leading to miss of true PLHEADER in that window. Considering the widest window is 33282 , the number of false PLHEADER could be conducted in the following equation.

$$
\text { Numbers }_{\text {false-PLHEADER }}=33282 \times\left(P_{-}^{3}+C_{3}^{2} P_{-}^{2}\left(1-P_{-}\right)\right)
$$

Even though $P_{\text {_ }}$ is rather small, i.e., $e^{-4}$, the number of false PLHEADER can be extraordinarily large after the multiplication of 33282 .

Some conclusions can be drawn from Fig. 10 and Fig. 11. The higher threshold to be set, the less number of false PLHEADER can be detected incorrectly, and the lower true PLHEADER synched probability can be reached for more PLHEADER miss.

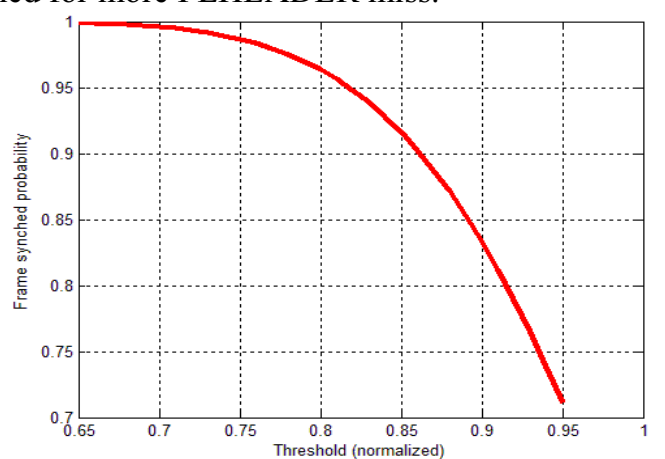

Figure 10. Theoretical frame synched probability versus threshold. 


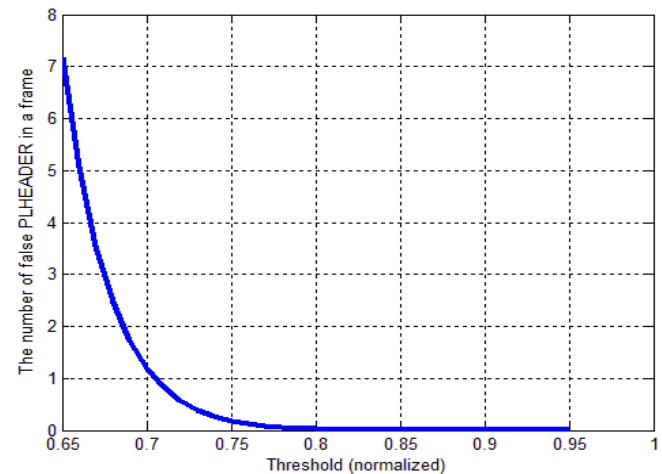

Figure 11. The number of false PLHEADER in a frame versus threshold.

\section{Optimizations and simplifications}

\subsection{Optimizations of differential detector}

The more features of the PLHEADER to be mined, the higher accuracy of the PLHEADER location can be achieved.

As described before, pilot blocks are inserted every 16 slots. Each pilot block is made up of 36 un-modulated symbols. To make full use of information in PLFRAME, differential detector can be more robust with the assistance of pilot block correlation. Pilot-symbolassisted differential correlation is analysed in [9], with three pilot blocks participating in differential correlation in trade-off between complexity and performance. However, this is not applicable to VCM, whose pilot blocks could be less than 3 . As can be seen in table 1, when information frame length is 16200 and modulation is 32APSK, there are only 2 pilot blocks in PLFRAME. To fit in with the various CODMOD in VCM, 2 pilot blocks are adopted in differential correlation as expressed in

$$
\wedge=\left|\sum_{k=1}^{25} r_{k} r_{k+1}^{*} C_{S O F k}^{*}+\sum_{m=1}^{45} R_{2 m-1} R_{2 m}^{*} C_{P L S C m}^{*}+\sum_{t=1}^{2} \sum_{h=1}^{35} p_{t, h} p_{t, h+1}^{*} C_{P i o t t, h}^{*}\right|
$$

where $r, R, C_{S O F}$ and $C_{P L S C}$ have the same meanings with equation (1), (2), (3). $p$ is the received symbol of pilot, $t$ is the index of pilot blocks, $h$ is the index of received symbol in a plot block, and star make represents complex conjugate.

$C_{\text {pilot }}$ is the coefficient of pilot block symbol, which can be obtained as

$$
C_{P i l o t t, h}=P_{t, h} P_{t, h+1}= \pm j, \pm 1(t=1,2, h=1,2, \ldots, 35),
$$

where $P$ denotes transmitted pilot symbols.

To validate the effectiveness of pilot block, simulation experiments are conducted under large frequency about $20 \%$ of transmission rate and extremely low SNR (-2.35dB) in Additive White Gaussian Noise (AWGN) channel. After regularized, PLHEADER and non-PLHEADER differential correlation distribution are more concentrated with the help of pilot, as shown in Fig. 12. The simulation presents a conclusion that differential detector shall be more robust with 2 pilot blocks participating in.

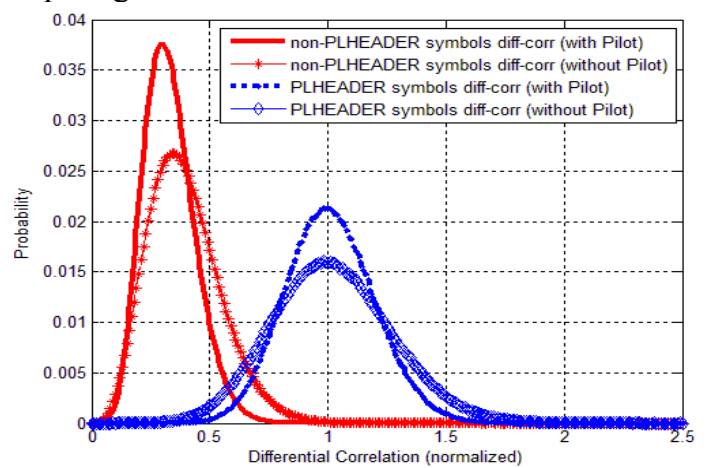

Figure 12. Differential correlation distribution in different detector.

\subsection{Simplifications of proposed algorithms}

In the proposed method, the set of window width shall cover all kinds of CODMODs, that is, frame synchronization controller shall search peak position in 16 windows in parallel. This also means that 16 suits of comparators and counters are in need, leading to high complexity. Some tricks can be done to simplify this nuisance in implementation.

First of all, there is a hidden fact that the length of FECREAME and pilot insertion usually remain constant, not varying from frame to frame. The length of the FECFRAME (64800 bits or 16200 bits) and pilot block insertion status depend on application area, meaning that we can get prior information about candidate windows type. Thus, in one satellite task, given the awareness of FECFRAME and pilot insertion status, only 4 types of window shall be considered, rather than 16 .

Moreover, to balance transmission throughput and implementation complexity, VCM system usually switches CODMODs 4 or 5 times during one satellite mission. Every switchover only leads to 3 or 4 frames loss in proposed method, which is negligible compared with millions of data frame in a mission. And, these frame losses in switchover can be eliminated by inserting 3 or 4 dummy frames after switching to another CODMOD.

\section{Simulation results}

\subsection{Threshold selecting in threshold-based algorithm}

To study the effect of threshold on frame synchronization rate, MATLAB simulations are conducted at fixed SNR = $-2.35 \mathrm{~dB}$ and frequency offset of $20 \%$ of transmission rate. Results are shown in Fig. 13. In the first stage, frame synchronization probability rises with increase of threshold for less false PLHEADERs. However, frame synchronization probability goes down in the second stage, due to more PLHEADER miss in high threshold. In VCM service, extremely bad channel shall be in consideration, thus threshold can be selected in range from 0.76 to 0.78 to get optimal performance. 


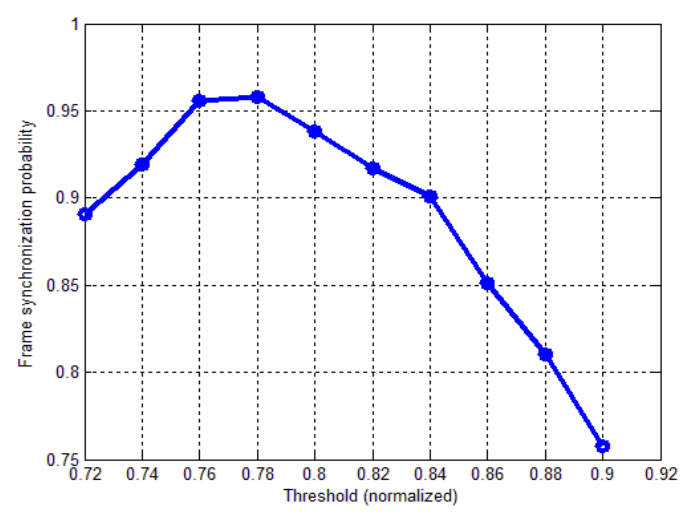

Figure 13. Frame synchronization probability versus threshold at $\mathrm{SNR}=-2.35 \mathrm{~dB}$.

\subsection{Performance comparison of different algorithms}

Experiments are conducted to investigate performance of proposed algorithm. With 20\% frequency offset, Fig. 14 shows the performance comparison for different SNR ranging in between $[-3,1]$. Threshold is set to 0.77 according to the result of Fig. 13 in threshold-based search algorithm. As Fig. 14 shows, peak search algorithm [7] and double peak search algorithm [8, 9] perform pretty well when SNRs are above $-0.5 \mathrm{~dB}$, and when channel condition turns to extremely bad, multiwindows threshold-based search algorithm shall prevail due to its novel scheme.

Actually, Multi-windows threshold-based search algorithm shares many similarities with double peak search algorithm in main procedure. The latter algorithm only considers two symbol locations of maximum differential correlation, while the former adds all locations whose differential correlations are above the threshold into inspection. When the channel is in good condition, e.g., the SNR is higher than $-0.5 \mathrm{~dB}$, both can achieve ideal performance with frame synchronization probability equaling to 1 . However, when SNR is lower than $-0.5 \mathrm{~dB}$, and noise strongly interferes useful signal, making PLHEADER symbol differential correlation far behind from the maximum. In conclusion, One peak and double peak search algorithm can be thought as special cases of threshold-based algorithm when threshold are set rather high.

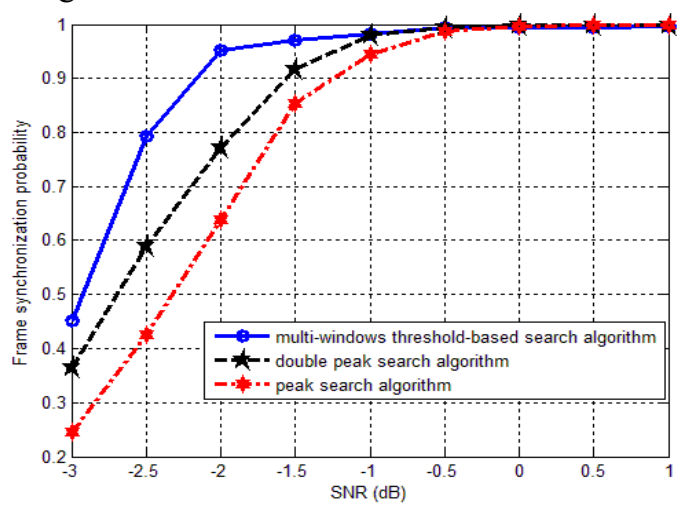

Figure 14. Frame synchronization probability versus threshold at $\mathrm{SNR}=-2.35 \mathrm{~dB}$.

\section{Conclusions}

Frame synchronization of DVB-S2 in VCM service is studied in this paper. Variability of frame coding and modulation makes it hard to achieve fast synchronization in VCM, thus, multi-windows are exploited. To decrease missed detection probability, threshold-based algorithm is proposed. Experimental results illustrate that our multiwindows threshold-based method is more robust and reliable than the existing methods, especially in the presence of large frequency offsets and low SNRs.

\section{References}

1. Digital video broadcasting (DVB); second generation framing structure, channel coding and modulation systems for broadcasting, interactive services, news gathering and other broadband satellite applications. (2005).

2. Estec ESA. VCM performance for LEO satellites at $26 \mathrm{GHz}$ a realistic assessment. (2010).

3. Zae Yong Choi and Yong H Lee. IEEE Trans. Commun, 50(7):1062-1065 (2002).

4. Giovanni E Corazza, Raffaella Pedone, and Marco Villanti. International Journal of Satellite Communications and Networking, 24(2):185-201 (2006).

5. Pansoo Kim, Giovanni Emanuele Corazza, Raffaella Pedone, Marco Villanti, Dae-Ig Chang, and DeockGil Oh. In Proc. IEEE Int. Conf. Wireless Communications and Networking, pp. 1183-1187 (2007).

6. Digital video broadcasting (DVB) user guidelines for the second generation system for broadcasting, interactive services, news gathering and other broadband satellite applications (dvb-s2). (2005).

7. Feng-Wen Sun, Yimin Jiang, and Lin-Nan Lee. International journal of satellite communications and networking, 22(3):319-339 (2004).

8. Li Q., Zeng X.Y., Wu c., et al, In Proc. IEEE Int. Conf. Circuits and Systems, pp. 956-959 (2008).

9. Yulong Zhang, Xubin Chen, Wenhua Fan, Jun Han, and Xiaoyang Zeng. In Proc. IEEE Int. Conf. Wireless Telecommunications Symposium, pp. 1-5 (2010).

10. Thomas A Sielicki, Jon Hamkins, and Denise Thorsen. In Proc. IEEE Int. Conf. Aerospace, pp. 17 (2013). 